\title{
Fluoride retention in infants living in fluoridated and non-fluoridated areas: effects of weaning
}

\author{
Fatemeh V. Zohoori ${ }^{1 *}$, Narges Omid ${ }^{1}$, Roy A. Sanderson ${ }^{2}$, Ruth A. Valentine ${ }^{3}$ and Anne Maguire ${ }^{3}$ \\ ${ }^{1}$ Health and Social Care Institute, Teesside University, Middlesbrough TS1 3BA, UK \\ ${ }^{2}$ Modelling, Evidence and Policy Research Group, School of Natural and Environmental Science, Newcastle University, \\ Newcastle upon Tyne NE2 4BW, UK \\ ${ }^{3}$ Centre for Oral Health Research, School of Dental Sciences, Newcastle University, Newcastle upon Tyne NE2 4BW, UK \\ (Submitted 21 March 2018 - Final revision received 18 September 2018 - Accepted 18 September 2018 - First published online 5 November 2018)
}

\section{Abstract}

Limited knowledge is available on total fluoride exposure, excretion and retention in infants, despite the first year of human life being the critical period for dental development and risk of dental fluorosis. This study investigated total daily fluoride intake (TDFI), excretion (TDFE) and retention (TDFR) in infants living in fluoridated and non-fluoridated water areas at pre- and post-weaning stages of development. Healthy infants, aged 0-12 months, were recruited and their TDFI ( $\mathrm{mg} / \mathrm{kg}$ body weight (BW) per $\mathrm{d}$ ), from diet and toothpaste ingestion, was assessed over a 3-d period using a dietary diary and tooth-brushing questionnaire. TDFE ( $\mathrm{mg} / \mathrm{kg} \mathrm{BW}$ per $\mathrm{d}$ ) was estimated by collecting 48-h urine and faeces. TDFR ( $\mathrm{mg} / \mathrm{kg}$ BW per $\mathrm{d}$ ) was estimated by subtracting TDFE from TDFI. A total of forty-seven infants completed the study: sixteen at pre-weaning and thirty-one at post-weaning stages, with a mean age of 3.4 and $10 \cdot 0$ months, respectively. TDFI was lower in the nonfluoridated area $(P<0.001)$ and at the pre-weaning stage $(P=0.002)$ but higher in formula-fed infants $(P<0 \cdot 001)$. TDFE was mainly affected by type of feeding, with higher excretion in formula-fed infants $(P<0 \cdot 001)$. TDFR was lower in the non-fluoridated area $(P<0 \cdot 001)$ and at the pre-weaning stage $(P<0.001)$ but higher in formula-fed infants $(P=0 \cdot 001)$. In conclusion, a relatively large proportion of fluoride intake is retained in the body in weaned infants. This is an important consideration in fluoride-based prevention programmes, with goals to maximise caries prevention while minimising the risk of dental fluorosis.

\section{Key words: Fluoride: Total intake: Urinary excretion: Faecal excretion: Retention}

Fluoride is mainly associated with calcified tissues in the body $^{(1)}$. It stimulates bone cell proliferation and increases new mineral deposition in cancellous bone ${ }^{(2)}$. Topical fluoride when applied to erupted teeth, for example through water fluoridation and tooth-brushing with a fluoridated dentifrice, can help reduce dental caries by almost $50 \%$ and is therefore a valuable public health measure to improve oral health ${ }^{(2)}$. Dental decay in primary and permanent teeth of children continues to be a major public health problem, with un-treated decay being the most common disease affecting humans worldwide ${ }^{(3)}$. Decay experience is higher in lower socio-economic groups: $83.4 \%$ of 5 -year-olds in lower-middle income countries have tooth decay compared with $49 \%$ of 5 -year-olds in higher-income countries ${ }^{(3)}$. This high prevalence of decay highlights the importance of primary fluoride-based prevention programmes, including fluoridated water, salt and milk. However, several studies ${ }^{(4,5)}$ have shown increased prevalence of dental fluorosis (mainly mild) in both fluoridated and non-fluoridated communities as a result of excessive systemic exposure to fluoride from multiple sources. Dental fluorosis is visible clinically as a white or brown 'mottling' of the tooth surface. The critical period for development of fluorosis in developing primary teeth is from 4 months in utero until 11 months of age ${ }^{(6)}$. Nonetheless, dental fluorosis in permanent maxillary central incisors is most likely to result from excessive fluoride exposure during the first 4 years of life, with the first 12 months being the most vulnerable period $^{(7)}$.

In children, the tolerable upper intake level (UL) of $0.1 \mathrm{mg} / \mathrm{kg}$ body weight (BW) per $\mathrm{d}$ has been suggested to minimise the risk of dental fluorosis ${ }^{(8,9)}$. Therefore, total daily fluoride intake (TDFI) at both individual and community levels should be considered when setting guidelines for fluoride use to maximise decay prevention and minimise dental fluorosis. Milk (breast milk or formula milk) is the main source of fluoride intake in infants aged $<6$ months old. However, introducing complementary feeding (i.e. weaning) and tooth-brushing, which

Abbreviations: BW, body weight; FFR, fractional fluoride retention; TDFE, total daily fluoride excretion; TDFI, total daily fluoride intake; TDFR, total daily fluoride retention.

* Corresponding author: Professor F. V. Zohoori, fax +44 1642 342770, email v.zohoori@tees.ac.uk 
usually starts at about 6 months of age, could have a marked impact on $\mathrm{TDFI}^{(10)}$

Several factors such as diet composition, age and body size could alter the rate of fluoride ingestion, absorption and retention $^{(1,11)}$. Therefore, it is important to quantify the bodyretained fluoride rather than only the absolute TDFI.

Knowledge about body fluoride retention originates from studies of healthy adults or laboratory animals, despite the first year of human life being the critical period for dental development and the risk of dental fluorosis. This is mainly because of the practical difficulties in recording dietary fluoride intake and collecting 24-h urine and faeces samples from infants and young children to estimate TDFI, total daily fluoride excretion (TDFE) and, consequently, body fluoride retention. Therefore, this study aimed to measure TDFI, TDFE and total daily fluoride retention (TDFR) in infants up to 12 months of age living in fluoridated and non-fluoridated water areas at pre- $(<6$ months old) and post-weaning (6-12 months old) stages of development.

\section{Methods}

This study was conducted according to the guidelines laid down in the Declaration of Helsinki, and all procedures involving human subjects/patients were approved by the School of Health and Social Care, Teesside University (\#079/13), and the Faculty of Medical Sciences, Newcastle University (\#00673/ 2013). Written informed consent was obtained from parental participants.

This observational study focused on parents of infants, aged 1-12 months, living in fluoridated and non-fluoridated water areas of North East England. The parents were recruited between January 2014 and February 2016 through major universities, nurseries and 'baby-group-centres' in the selected areas. The participants were visited at their homes when weights of infants were measured, using a calibrated digital baby scale (Learning Curve Brands Inc.), and samples/data were collected. The infants were included if they were healthy, had lived continuously in the selected areas and were fed exclusively either breast milk or formula milk.

\section{Estimation of total daily fluoride intake}

A food diary was given to each parent with instructions on how to complete it on three consecutive days for their infant. Each parent was interviewed on the 4th day to ensure that all food/ drink items consumed by their infant had been recorded. Samples of breast and/or formula milk, consumed by all infants, as well as samples of all home-made food and drink consumed by weaned infants during the 3 -d dietary assessment, were collected for fluoride analysis. Information on fluoride concentration in commercially available baby food/drinks was taken from an existing fluoride database ${ }^{(12)}$. Dietary fluoride intake $(\mu \mathrm{g} / \mathrm{d})$ for each infant was estimated by multiplying the average intake of each item $(\mathrm{g} / \mathrm{d})$ by its fluoride concentration $(\mu \mathrm{g} / \mathrm{g})$. In breast-fed infants, consumption of breast milk was quantified from reported energy requirements, by age and sex ${ }^{(13)}$, and the average energetic density of human milk of $651 \mathrm{kcal} / \mathrm{l}(2724 \mathrm{~kJ} / \mathrm{l})^{(14)}$.

A parental oral hygiene questionnaire with interview was used to obtain information on the infant's tooth-brushing habits, and the frequency of cleaning/brushing, type and amount of any dentifrice used were recorded. The amount of fluoride ingested through brushing was estimated from the weight and frequency of dentifrice use, assuming that almost all dentifrice used in brushing is swallowed by an infant at this age.

No infant used fluoride supplements; therefore, the TDFI for each infant was calculated by combining fluoride intake from diet and dentifrice use.

\section{Estimation of total daily fluoride excretion}

Parents were supplied with specially designed pads (Uricol; Sterisets) for use inside the child's diaper (nappy) ${ }^{(15)}$ and instructed on how to collect and store wet (urine) and/or soiled/wet (urine and faeces) pads/diapers over $48 \mathrm{~h}$. For wet pads/diapers, parents were asked to withdraw a 5-ml urine sample from the pad, using the plastic syringe provided, and transfer the sample to a labelled container. The collected samples were weighed to measure the weight of urine and/or faeces excreted. The faeces were removed from the pads using a disposable wooden spatula, transferred into a labelled bag and stored at $-20^{\circ} \mathrm{C}$ before fluoride analysis.

To quantify the fluoride content of each individual excrement ( $\mathrm{mg} / \mathrm{sample}$ ), its measured fluoride concentration $(\mu \mathrm{g} / \mathrm{g})$ was multiplied by the corresponding weight of excreta (i.e. either urine or combined urine and faeces; g). The TDFE for each infant was then computed by summing the calculated fluoride content of individual excreta.

\section{Fluoride analysis}

Sample analyses were conducted from February 2016 to December 2017. Fluoride concentrations in home-prepared food and milk samples consumed by infants and in excreted faeces were measured by the acid-diffusion method, with fluoride assay of urine, non-milk-based drink and water samples undertaken using a direct method with fluoride-ionselective electrode (Model 9409; Orion) and meter (Model 900A; Orion) after addition of TISAB-III (total ionic strength adjustment buffer), with the sample-TISAB ratio of 10:1 $(\mathrm{v} / \mathrm{v})^{(16)}$

Analytical quality control (AQC) was undertaken by (i) conducting incurred sample re-analysis (ISR) and (ii) using a certified reference material (CRM). ISR ${ }^{(17)}$ was performed by reanalysing $5 \%$ of samples in separate runs on different days from when the analysis was performed. The percentage difference between the results was determined using the following formula: $(($ repeat-original $) /$ mean $) \times 100^{(17)}$. In addition, a paired $t$ test was used to test for the difference between the original and the re-analysed (repeat) data. A certified reference 'urine fluoride' sample (\#PC-U-F1703; Institut National de Santé Publique du Québec) was used as the CRM and tested blind by the study technician. 


\section{Data analysis}

TDFR (mg/d) was calculated by subtracting TDFE (mg/d) from TDFI $(\mathrm{mg} / \mathrm{d})$ for each infant, and the relevant variables were normalised by BW (mg/kg BW per d). Fractional fluoride retention (FFR) was estimated as the ratio between TDFR and TDFI.

\section{Statistical analysis}

Sample size. A power analysis was undertaken using $\mathrm{R}$ to estimate appropriate sample size based on results from a previous study of TDFI by Zohoori et $a l{ }^{(10)}$, which used nineteen infants per group. It was estimated that 3.9 individuals per group would be needed for $80 \%$ power.

ANOVA was used to evaluate the effects of weaning stage (pre- $v$. post-weaning), area of residency (fluoridated $v$. nonfluoridated) and type of feeding (breast milk $v$. formula milk) on TDFI, TDFE and TDFR. Initially, the area of residency, weaning stage and type of feeding were used as predictors, plus all interaction terms; non-significant interaction terms were sequentially removed, along with main effects where necessary, until the best minimal model was identified. Mean and $95 \%$ CI of the differences were calculated for the terms in the minimal model. Effect sizes were calculated as $\eta^{2(18)}$, which are more useful than the more widely used measure of Cohen's $d$ in an ANOVA design such as our experiment, where feasibility of recruitment to the study was a limiting factor. $\eta^{2}$ can be interpreted as the proportion of total variability in the response variable that can be accounted by the explanatory variable ${ }^{(19)}$. Cohen ${ }^{(20)}$ provided approximate $\eta^{2}$ benchmarks for 'small' (0.01), 'medium' (0.06) and 'large' $(0.14$ or greater $)$ effect sizes. As the effect size is basically a way of quantifying the absolute size of the difference between two groups, the larger the effect size, the more reliable the difference. A result is more likely to be biologically meaningful if it is both statistically significant and has a large effect size.

Long-term relationships between age (predictor) and the response variables (i) TDFI, (ii) TDFE and (iii) TDFR were evaluated with the aid of standard linear regressions and Pearson's correlation coefficients.

\section{Results}

Initially, seventy-seven parents expressed interest to participate in the study, of whom twenty-eight subsequently did not sign the consent form owing to the extent of the required commitment from them (e.g. collection of urine/faeces samples). Two infants were excluded, as they were receiving combined feeding (breast and formula).

The majority (64\%) of infants were male. Table 1 presents mean (SD) age and weight of the forty-seven infants, who completed the study, per group. The study managed to recruit more than four infants per group for all but two exposure combinations: un-weaned breast-fed infants living in a nonfluoridated area $(n 2)$ and weaned formula-fed infants living in a non-fluoridated area ( $n$ 3). The overall mean age and weight of the sixteen un-weaned infants were 3.4 (SD 1.1) months and $6 \cdot 3$ (SD 1.1) kg, respectively, and of the thirty-one weaned infants the mean age and weight were 10.0 (SD 1.2) months and 8.9 (sD $0 \cdot 8) \mathrm{kg}$, respectively.

In total, 416 wet (urine) and 245 soiled/wet (urine and faeces) diapers were collected from all infants; that is on average seven diapers/d per child. In addition, 124 breast milk samples and 138 water samples, as well as ninety-four food diaries, were collected.

Regarding the AQC, the mean ISR was 11 (SD 9)\%, well below the recommended ISR acceptance criteria (within $20 \%$ for small molecules and $30 \%$ for macromolecules) ${ }^{(17)}$. There was no statistically significant difference in fluoride concentration between original and repeat analyses (mean difference: $0.011 \mathrm{mg} / \mathrm{l} ; 95 \% \mathrm{CI}-0.019,0.041 \mathrm{mg} / \mathrm{l}$ ). The measured fluoride concentrations of the original and repeat CRM samples were 0.232 and $0.235 \mathrm{mg} / \mathrm{l}$, respectively, which were within the acceptable range (certified target value: $0.283 \mathrm{mg} / \mathrm{l}$; and acceptable range: $0 \cdot 182-0 \cdot 384 \mathrm{mg} / \mathrm{l})$.

Fig. 1 shows weight-normalised TDFI, TDFE and TDFR for all infants by weaning stage, type of feeding and area of residency. The mean TDFI of all infants was $<0 \cdot 1 \mathrm{mg} / \mathrm{kg} \mathrm{BW}$ per $\mathrm{d}$. TDFR was negative in the breast-fed infants living in fluoridated $(-0.005 \mathrm{mg} / \mathrm{kg} \mathrm{BW}$ per d) and non-fluoridated $(-0.003 \mathrm{mg} / \mathrm{kg}$ BW per d) areas, as well as in the un-weaned formula-fed infants living in the non-fluoridated area $(-0.007 \mathrm{mg} / \mathrm{kg}$ BW per d), whereas the highest mean TDFR $(0.061 \mathrm{mg} / \mathrm{kg} \mathrm{BW}$ per d)

Table 1. Infant data stratified by weaning stage, type of feeding and area of residency (Mean values and standard deviations)

\begin{tabular}{|c|c|c|c|c|c|c|c|c|c|c|c|c|c|c|c|}
\hline \multirow[b]{3}{*}{ Infant } & \multicolumn{5}{|c|}{ 1-6 month-old infants } & \multicolumn{5}{|c|}{ 6-12 month-old infants } & \multicolumn{5}{|c|}{ All infants (1-12 months) } \\
\hline & \multirow[b]{2}{*}{ No. } & \multicolumn{2}{|c|}{ Age (months) } & \multicolumn{2}{|c|}{ Weight (kg) } & \multirow[b]{2}{*}{ No. } & \multicolumn{2}{|c|}{ Age (months) } & \multicolumn{2}{|c|}{ Weight (kg) } & \multirow[b]{2}{*}{ No. } & \multicolumn{2}{|c|}{ Age (months) } & \multicolumn{2}{|c|}{ Weight $(\mathrm{kg})$} \\
\hline & & Mean & SD & Mean & $\mathrm{SD}$ & & Mean & SD & Mean & SD & & Mean & SD & Mean & SD \\
\hline All breast-fed and formula-fed & 16 & $3 \cdot 4$ & $1 \cdot 1$ & $6 \cdot 3$ & $1 \cdot 1$ & 31 & $10 \cdot 0$ & $1 \cdot 2$ & 8.9 & 0.8 & 47 & $7 \cdot 8$ & $3 \cdot 4$ & $8 \cdot 0$ & 1.6 \\
\hline Breast-fed, all & 7 & $3 \cdot 8$ & $1 \cdot 0$ & $6 \cdot 4$ & 1.6 & 16 & 1.02 & $1 \cdot 2$ & 8.7 & 0.8 & 23 & $8 \cdot 3$ & $3 \cdot 2$ & $8 \cdot 0$ & 1.5 \\
\hline Breast-fed, FA & 5 & 4.0 & $1 \cdot 2$ & $6 \cdot 6$ & 1.8 & 8 & 9.9 & $1 \cdot 2$ & 8.5 & 0.7 & 13 & $7 \cdot 6$ & $3 \cdot 2$ & $7 \cdot 8$ & 1.5 \\
\hline Breast-fed, NFA & 2 & 3.4 & 0.5 & 5.9 & 0.9 & 8 & 10.5 & $1 \cdot 1$ & 9.0 & 0.9 & 10 & $9 \cdot 1$ & $3 \cdot 2$ & 8.4 & 1.6 \\
\hline Formula-fed, all & 9 & $3 \cdot 1$ & $1 \cdot 2$ & $6 \cdot 2$ & 0.8 & 15 & 9.8 & $1 \cdot 2$ & 9.2 & 0.8 & 24 & $7 \cdot 3$ & 3.5 & 8.0 & 1.7 \\
\hline Formula-fed, FA & 4 & 3.4 & 1.0 & 6.5 & 0.4 & 12 & $10 \cdot 0$ & 1.1 & 9.2 & 0.8 & 16 & 8.4 & $3 \cdot 1$ & 8.5 & 1.4 \\
\hline Formula-fed, NFA & 5 & $2 \cdot 8$ & 1.4 & $6 \cdot 0$ & 0.9 & 3 & 8.8 & 1.3 & $9 \cdot 1$ & 1.0 & 8 & $5 \cdot 1$ & 3.3 & $7 \cdot 1$ & 1.9 \\
\hline
\end{tabular}

FA, fluoridated area (fluoride concentration of tap water: 0.86 (SD 0.23) mg/l); NFA, non-fluoridated area (fluoride concentration of tap water: 0.12 (SD 0.09 ) mg/l). 
Fig. 1. Total fluoride intake, excretion and retention ( $\mathrm{mg} / \mathrm{kg}$ body weight $(\mathrm{BW})$ per $\mathrm{d}$ ) stratified by weaning stage (pre- $v$. post-weaning), type of feeding (breast- $v$. formula-milk) and area of residency (fluoridated (F) $v$. non-fluoridated (NF)) in infants younger than 12 months. Fluoridated area (0.86 parts per million (ppm) fluoride); non-fluoridated area $(0.12 \mathrm{ppm}$ fluoride); unweaned infants ( $<6$ months old); weaned infants (6-12 months old). 7 , Total daily fluoride intake (mg/kg BW per d); $\square$, total daily fluoride excretion (mg/kg BW per d); $\square$, total daily fluoride retention (mg/kg BW per d). Values are means, with $95 \%$ confidence intervals represented by vertical bars.

Table 2. Summary of results of best sequential linear model, with weight-normalised total daily fluoride intake, excretion and retention (as the response)*

(Mean values and $95 \%$ confidence intervals)

\begin{tabular}{|c|c|c|c|c|}
\hline \multirow[b]{2}{*}{ Source } & \multicolumn{3}{|c|}{ Differences between variables } & \multirow[b]{2}{*}{$\eta^{2}$} \\
\hline & Mean & $95 \% \mathrm{Cl}$ & $P$ & \\
\hline \multicolumn{5}{|l|}{ Total daily fluoride intake (mg/kg BW per d) } \\
\hline Area of residency (NFA $v . F A)$ & -0.027 & $-0.043,-0.012$ & $<0.001$ & 0.001 \\
\hline Weaning stage (pre-weaning $v$. post-weaning) & -0.027 & $-0.043,-0.011$ & 0.002 & 0.068 \\
\hline Type of feeding (FF $v . \mathrm{BF}$ ) & 0.037 & $0.022,0.052$ & $<0.001$ & 0.308 \\
\hline Area of residency $\times$ type of feeding interaction & & & & 0.087 \\
\hline 'FA:FF' v. 'FA:BF' & 0.056 & $0.030,0.081$ & $<0.001$ & \\
\hline 'FA:FF' v. 'NFA:BF' & 0.056 & $0.028,0.084$ & $<0.001$ & \\
\hline 'NFA:FF' v. 'FA:FF' & -0.048 & $-0.078,-0.018$ & $<0.001$ & \\
\hline \multicolumn{5}{|l|}{ Total daily fluoride excretion (mg/kg BW per d) } \\
\hline Weaning stage (pre-weaning v. post-weaning) & 0.006 & $-0.001,0.013$ & 0.085 & 0.004 \\
\hline Type of feeding (FF v. BF) & 0.017 & $0.011,0.023$ & $<0.001$ & 0.145 \\
\hline Weaning stage $\times$ type of feeding interaction & & & & 0.055 \\
\hline 'Post-weaning: FF' v. 'post-weaning:BF' & 0.013 & $0.002,0.023$ & 0.010 & \\
\hline 'Pre-weaning: FF' $v$. 'post-weaning:BF' & 0.023 & $0.011,0.035$ & $<0.001$ & \\
\hline 'Post-weaning: FF' v. 'pre-weaning:BF' & 0.015 & $0.002,0.028$ & 0.016 & \\
\hline 'Pre-weaning: FF' v. 'pre-weaning:BF' & 0.026 & $0.012,0.040$ & $<0.001$ & \\
\hline \multicolumn{5}{|l|}{ Total daily fluoride retention $(\mathrm{mg} / \mathrm{kg} \mathrm{BW}$ per d) } \\
\hline Area of residency (NFA $v$. FA) & -0.024 & $-0.036,-0.012$ & $<0.001$ & 0.0003 \\
\hline Weaning stage (pre-weaning $v$. post-weaning) & -0.033 & $-0.045,-0.020$ & $<0.001$ & 0.168 \\
\hline Type of feeding (FF $v . \mathrm{BF}$ ) & 0.020 & $0.008,0.032$ & 0.001 & 0.210 \\
\hline Area of residency $\times$ type of feeding interaction & & & & 0.104 \\
\hline 'FA: FF' v. 'FA: BF' & 0.036 & $0.016,0.056$ & $<0.001$ & \\
\hline 'FA: FF' v. 'NFA: BF' & 0.038 & $0.016,0.060$ & $<0.001$ & \\
\hline 'NFA: FF' v. 'FA: FF' & -0.042 & $-0.066,-0.090$ & $<0.001$ & \\
\hline
\end{tabular}

NFA, non-fluoridated area (fluoride concentration of tap water: 0.12 (sD 0.09) $\mathrm{mg} / \mathrm{l}$ ); FA, fluoridated area (fluoride concentration of tap water: 0.86 (sD 0.23) $\mathrm{mg} / \mathrm{l})$; FF, formula-fed; BF, breast-fed.

* The non-significant variables are not presented in the table.

was observed in the weaned formula-fed infants living in the fluoridated area. The best linear models (Table 2) indicated that TDFI was significantly higher in fluoridated areas $(P<0 \cdot 001)$, in formula-fed infants $(P<0.001)$ and post weaning $(P=0.002)$. There was also a strong interaction between the area of residency and type of feeding, with TDFI being significantly higher $(P<0.001)$ where infants were both formula-fed and residing in fluoridated areas. However, the observed small, medium and large effect sizes for area of residency $\left(\eta^{2}=0.001\right)$, weaning stage $\left(\eta^{2}=0.068\right)$ and type of feeding $\left(\eta^{2}=0.308\right)$, 
respectively, clearly imply that type of feeding was the main factor affecting TDFI, whereas weaning stage was the second most important factor.

TDFE was significantly $(P<0.001)$ lower in breast-fed compared with formula-fed infants (with a large effect size; $\eta^{2}=0.145$ ), and there was an interaction between weaning stage and type of feeding, with TDFE being significantly $(P<0.001)$ lower in un-weaned breast-fed infants. As the area of residency had no effect on TDFE, either as a main effect or any interaction term, it was omitted from the final model.

TDFR was significantly higher in fluoridated areas $(P<0 \cdot 001)$ and in weaned infants $(P<0.001)$ but lower in breast-fed infants $(P=0.001)$. There was a significant interaction between area of residency and type of feeding $(P<0 \cdot 001)$, with TDFR being lower than would otherwise be expected, where infants were breast-fed and lived in non-fluoridated areas. The large effect sizes detected for both weaning stage $\left(\eta^{2}=0 \cdot 168\right)$ and type of feeding $\left(\eta^{2}=0 \cdot 210\right)$ denote the significance of these two factors in TDFR.

Mean body-retained fluoride as a percentage of TDFI (i.e. FFR) for all infants is shown in Fig. 2. At pre-weaning, the FFR was only positive in formula-fed infants living in the fluoridated area. The overall mean FFR for all thirty-one infants, at the postweaning stage, was 65 (sE 3)\%.

The linear relationships between age and weight-normalised TDFI, TDFE and TDFR for breast-fed infants and formula-fed infants are presented in Fig. 3 and 4, respectively. In breast-fed infants (Fig. 3), the statistically significant positive correlation between age and TDFI $(\mathrm{mg} / \mathrm{kg}$ BW per $\mathrm{d})$ was moderate $(\rho=0.51, P=0.012)$, but it was strong $(\rho=0.63, P=0.001)$ between age and TDFR. In formula-fed infants (Fig. 4), there was a statistically significant positive moderate correlation between age and TDFI ( $\rho=0 \cdot 41, P=0 \cdot 044)$, as well as between age and TDFR $(\rho=0.56, P=0.004)$.

\section{Discussion}

Clinically, the affinity of fluoride for bone and teeth is high, and fluoroapatite crystals that form at the surface of bone or enamel are important for tooth enamel hardness and bone mineral matrix stability ${ }^{(21)}$. Uptake of fluoride during the pre-eruptive stage of enamel formation is dentally important owing to the increased risk of dental fluorosis development if systemic ingestion of fluoride is chronically excessive in early infancy. Therefore, for fluoridation policy decision-making and informing caries prevention programmes, continued assessment of total fluoride exposure in young children has been recommended ${ }^{(22,23)}$

The mean TDFI of all infants, in the present study, was below the UL of $0.1 \mathrm{mg} / \mathrm{kg} \mathrm{BW}$ per $\mathrm{d}^{(8,9)}$. Moreover, the present study showed that, in infants, the TDFI was overwhelmingly affected by type of feeding, whereas the effects of weaning stage and area of residency were moderate (see interaction terms in Table 2). At the pre-weaning stage, milk (either breast milk or formula milk) was the sole source of fluoride intake for these infants. The mean TDFI of infants $<6$ months old exclusively breast-fed and living in both fluoridated and non-fluoridated areas was almost negligible (Fig. 1; 0.005 and $0.001 \mathrm{mg} / \mathrm{kg} \mathrm{BW}$ per d, respectively). Generally, the fluoride concentration of breast milk is very low $(<0.02 \mu \mathrm{g} /$ $\mathrm{ml}$ ), with no significant difference in breast-milk fluoride concentration found between mothers living in fluoridated and nonfluoridated areas ${ }^{(24)}$, owing to the limited transfer of fluoride from plasma to breast milk ${ }^{(25)}$. Breast-fed infants typically receive up to a maximum 'dose' of $0.2 \%$ of the maternal fluoride intake ${ }^{(26)}$.

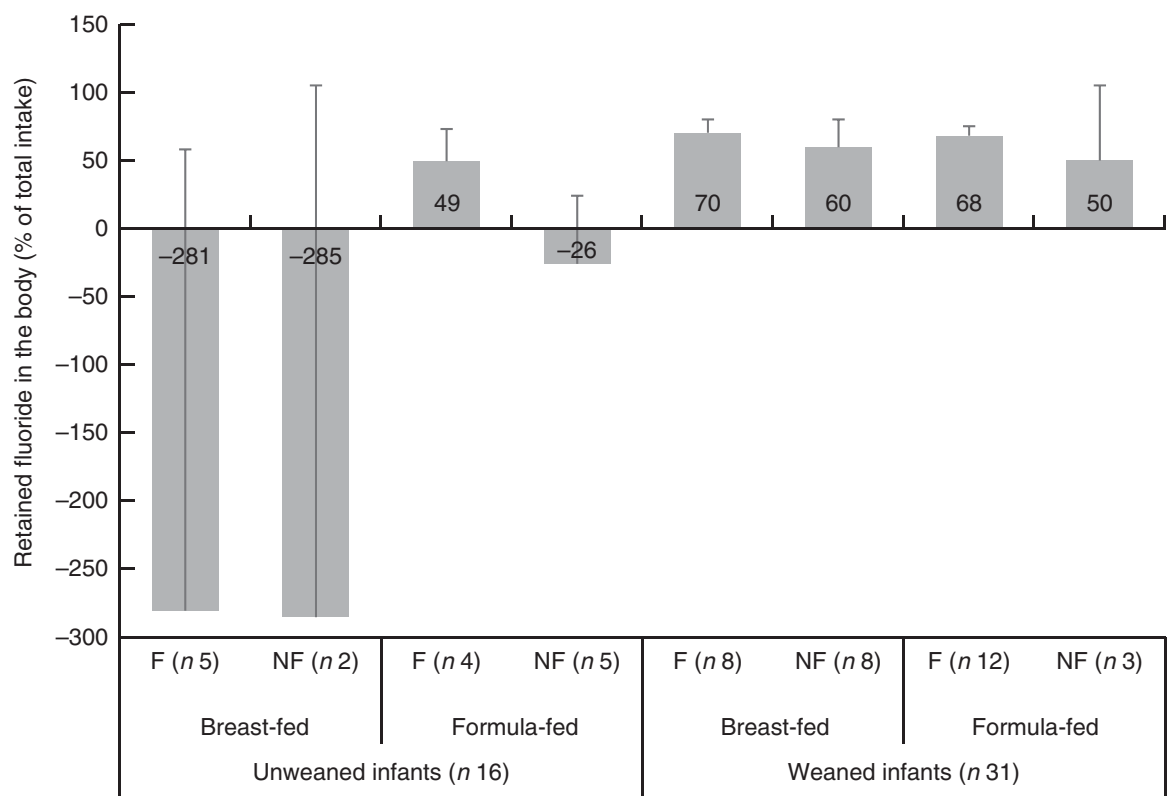

Fig. 2. Retained fluoride in the body as a percentage of total fluoride intake (fractional fluoride retention), stratified by weaning stage (pre- $v$. post-weaning), type of feeding (breast- $v$. formula-milk) and area of residency (fluoridated (F) $v$. non-fluoridated (NF)) in infants younger than 12 months old. Fluoridated area ( 0.86 parts per million (ppm) fluoride); non-fluoridated area ( $0.12 \mathrm{ppm}$ fluoride); unweaned infants (<6 months old); weaned infants (6-12 months old). Values are means, with $95 \%$ confidence intervals represented by vertical bars. 


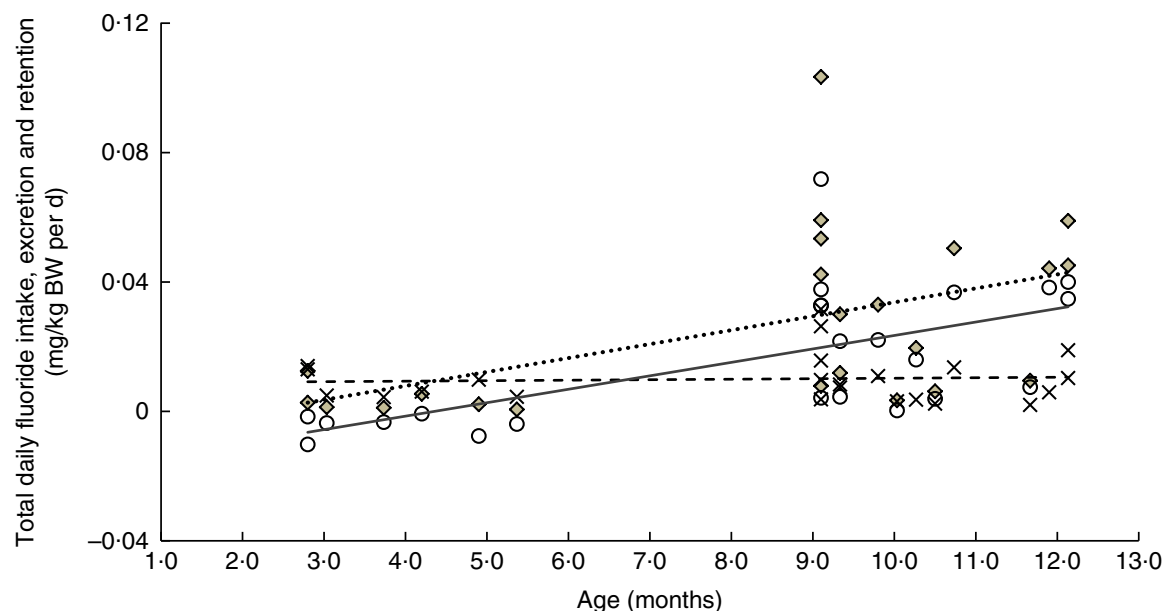

Fig. 3. Relationship between age- and weight-normalised - (i) total daily fluoride intake (TDFI), (ii) total daily fluoride excretion (TDFE) and (iii) total daily fluoride retention (TDFR) in breast-fed infants $(n$ 23). TDFI $(\diamond, \cdots \cdots, \mathrm{mg} / \mathrm{kg}$ body weight $(\mathrm{BW})$ per d) $=-0.009+0.004$ (age (months)) $(\rho=0.51, P=0.012)$, TDFE $(X,---\cdot$, $\mathrm{mg} / \mathrm{kg} \mathrm{BW}$ per d $)=0.009+0$ (age (months)) $(\rho=0.06, P=0.774)$ and TDFR $(\mathrm{O}, \longrightarrow, \mathrm{mg} / \mathrm{kg} \mathrm{BW} \mathrm{per} \mathrm{d})=-0.018+0.004($ age $($ months $))(\rho=0.63, P=0.001)$.

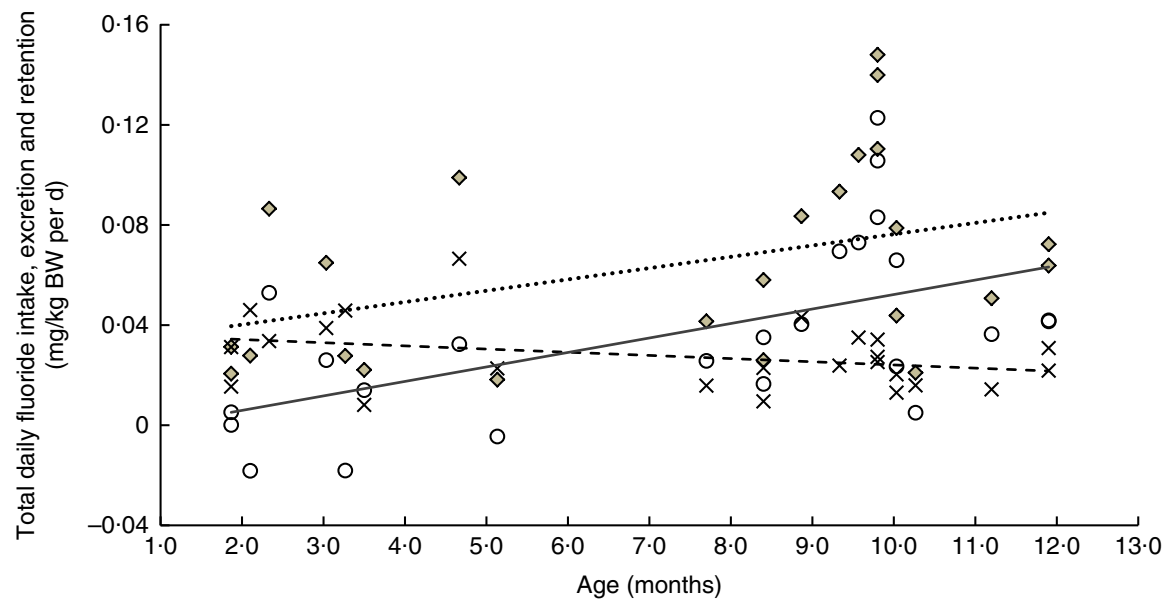

Fig. 4. Relationship between age- and weight-normalised (i) total daily fluoride intake (TDFI), (ii) total daily fluoride excretion (TDFE) and (iii) total daily fluoride retention (TDFR) in formula-fed infants $(n 24)$. TDFI $(\diamond, \cdots \cdots, \mathrm{mg} / \mathrm{kg}$ body weight (BW) per d) $=-0.031+0.005$ (age (months)) $(\rho=0.41, P=0.044)$, TDFE $(X,---$, $\mathrm{mg} / \mathrm{kg} \mathrm{BW}$ per $\mathrm{d})=0.037-0.001$ (age (months)) $(\rho=-0.32, P=0.122)$, TDFR $(\mathrm{O},-, \mathrm{mg} / \mathrm{kg} \mathrm{BW}$ per $\mathrm{d})=-0.006+0.006($ age $($ months $))(\rho=0.56, P=0.004)$.

A considerable difference in fluoride intake between exclusively breast-fed and formula-fed infants has also been reported for US infants aged 2-6 months living in a fluoridated area with $1 \mathrm{mg}$ fluoride/l in drinking water: $0.001 \mathrm{mg} / \mathrm{kg} \mathrm{BW}$ per $\mathrm{d}$ in the three breast-fed $v \cdot 0 \cdot 141 \mathrm{mg} / \mathrm{kg}$ BW per $\mathrm{d}$ in the five formula-fed infants $^{(27)}$

A significant increase in the weight-corrected TDFI with increasing age was observed in both breast-fed (Fig. 3) and formula-fed (Fig. 4) infants. This increase is likely to be due to introduction of weaning food/drinks containing fluoride and post-weaning fluoridated dentifrice use (i.e. in 6-12 month-olds). A positive trend between TDFI and age has also been reported for infants aged 1-12 months, living in non-fluoridated areas in England $^{(10)}$. However, an inverse relationship between age and TDFI has been reported for 1-12 month-old English infants ${ }^{(10)}$ living in a fluoridated area $\left(0.97 \mathrm{mg}\right.$ fluoride/l) and US (Iowa) ${ }^{(28)}$ infants living in areas with fluoride water concentrations of $<0.3$ $2.0 \mathrm{mg} / \mathrm{l}$. The between-studies differences in the age-TDFI relationship could be due to differences in age, feeding pattern and oral hygiene habits of the studied infants. For example, in the UK study ${ }^{(10)}$, more than $70 \%$ of infants were younger than 6 months, with $10 \%$ being exclusively breast-fed, compared with 34 and $15 \%$, respectively, in the present study.

Despite the significant increase in TDFI with age, the TDFE remained almost constant with increasing age (Fig. 3 and 4). This finding can be explained by the low renal function found in infancy, even when the appropriate correction is made for the small body size ${ }^{(29)}$. After birth, maturation is still a process involving all organs including the kidneys. Effective renal plasma flow increases from $83 \mathrm{ml} / \mathrm{min}$ per $1.73 \mathrm{~m}^{2}$ in full-term infants to an adult rate of $650 \mathrm{ml} / \mathrm{min}$ per $1.73 \mathrm{~m}^{2}$ at approximately 2 years of age and glomerular filtration rate increases from $40 \mathrm{ml} / \mathrm{min}$ per $1.73 \mathrm{~m}^{2}$ at birth to normal adult values of $100-125 \mathrm{ml} / \mathrm{min}$ per $1.73 \mathrm{~m}^{2}$ by 2 years of age ${ }^{(30)}$. Consequently, infants and toddlers have limited capacity for metabolism and elimination of different drugs and elements such as 
fluoride from the body, as their renal function only reaches full capacity by the age of 2 years.

The TDFE was largely influenced by type of feeding, with significantly lower TDFE in the breast-fed compared with formula-fed infants (Fig. 1). However, the effect of type of feeding on TDFE was moderated by the weaning stage (Table 2). Although lower TDFE in the breast-fed infants could be explained largely by their lower TDFI, as observed in the study, differences in the degree of fluoride absorption between breast- and formula-fed infants should also be considered. The systemic bioavailability of fluoride from different sources is influenced by the presence or absence of other nutrients; for example, $\mathrm{Al}, \mathrm{Ca}, \mathrm{Mg}$ and chloride increase faecal fluoride excretion, whereas dietary fats improve fluoride absorption ${ }^{(1)}$. Breast milk contains more fat but fewer minerals than infant formula; therefore, faecal fluoride excretion (and consequently TDFR) would be expected to be lower in breast-fed infants.

The TDFR was largely influenced by weaning stage and type of feeding (Table 2). In un-weaned infants ( $<6$ month-olds), those breast-fed living in both fluoridated- and non-fluoridated areas, as well as those formula-fed living in the non-fluoridated area, had a negative fluoride retention (Fig. 2) indicating a negative fluoride balance (i.e. fluoride excretion $>$ fluoride intake). A negative fluoride balance has also been reported for five 2-6 month-old breast-fed US infants living in a fluoridated area ${ }^{(27)}$. Fluoride balance in infants can depend on plasma fluoride concentration and the concurrent fluoride concentration in the rapidly exchangeable fluoride pool of bone; that is, a fall in plasma fluoride concentration results in a net migration of fluoride from bone to plasma and vice versa. Fluoride can cross the placenta and accumulate in fetal calcified tissues ${ }^{(31)}$. However, when fluoride intake of very young infants is lower than that needed to maintain the in utero plasma fluoride concentration (e.g. in breast-fed infants), mobilisation of fluoride from calcified tissues into plasma occurs, which results in fluoride excretion that exceeds the levels of fluoride intake (i.e. negative balance) ${ }^{(1)}$. However, the study found (Fig. 2) no statistically significant differences in the proportion of TDFI, which was retained in the body of weaned infants (6-12 montholds), indicating the effect of weaning (i.e. eating other sources of food other than just milk) on FFR. For example, the bioavailability of fluoride from infant milk formula reconstituted with water is $65 \%$, whereas a mixed diet may reduce absorption of fluoride by $47 \%{ }^{(32,33)}$.

The mean FFR of $49 \%$ (Fig. 2) for the 2-6 month-old formulafed infants living in the fluoridated area was lower than the corresponding figure of $58 \%$ calculated for 2-6 month-old formula-fed US infants living in a fluoridated area ${ }^{(27)}$. In addition, overall mean FFR in the thirty-one (65\%) infants aged 6-12 months was higher than the corresponding value of $50 \%$ reported for six US infants aged 6-12 months living in a fluoridated area ${ }^{(34)}$ but in agreement with the $65 \%$ reported for 212 children aged $<7$ years ${ }^{(35)}$.

\section{Limitations}

The possible limitations of this study are the sample size (though only in two of the eight sub-groups), convenience sampling strategy and non-equally sized subgroups. Despite the prolonged recruitment efforts, the poor recruitment rate was mainly due to the high burden of collecting detailed exposure data over $3 \mathrm{~d}$, as well as samples of urine and faeces over $2 \mathrm{~d}$ from infants by parents. However, the number of infants in the present study ( $n$ 47) was substantially higher than those recruited in similar studies: 'five breast-fed and five bottle-fed infants' in a fluoride balance study ${ }^{(27)}$ and 'two breast-fed and fifteen formula-fed infants (eleven females and six males)' in a fluoride pharmacokinetic study ${ }^{(36)}$

The main limitation of this study is that urine and faeces were not collected separately owing to ethical issues related to more invasive methods of urine and faeces collection, such as the use of metabolic beds, needed to separate collection of these types of samples ${ }^{(36)}$. Therefore, no conclusions can be drawn from this study about the effect of type of feeding (breast $v$. formula) on the degree of excretion of the ingested fluoride (i.e. faecal excretion) or the absorbed fluoride (i.e. urinary excretion).

\section{Conclusion}

The data showed that the type of feeding is the single most important factor in fluoride intake, exertion and retention in infants, although the area of residency also has smaller but significant impacts on fluoride intake and retention. The data have confirmed that fluoride intakes of exclusively breast-fed infants, irrespective of area of residency, as well as un-weaned formula-fed infants living in non-fluoridated areas, are so low that they result in a negative fluoride balance (i.e. mobilisation of fluoride from calcified tissues). However, in weaned infants, a relatively large proportion of fluoride intake is retained in the body (i.e. deposited in calcifying tissues). It is important that this is taken into consideration when implementing fluoride-based prevention programmes for young children. The goals for these interventions should be to maximise caries prevention while minimising dental fluorosis risk. Current evidence is that the main caries preventive effects of fluoride are due to its local topical effects at the tooth surface, whereas dental fluorosis results from chronic excessive systemic ingestion of fluoride from different sources during crucial periods of tooth development. In view of this, recommendations from the American Dental Association ${ }^{(37)}$ such as 'continue breast-feeding throughout the first year of life' and 'use (infant) formula reconstituted with water that is either fluoride-free or has low concentrations of fluoride when the potential risk for enamel fluorosis is a concern' remain key to harnessing the caries benefits of fluoride while minimising risk of fluorosis.

\section{Acknowledgements}

The authors thank the parents for their participation and cooperation.

The present study was funded by a grant from the Organix Foundation, gratefully acknowledged, which was disclosed to the study participants. The views expressed in this paper are those of the authors and not those of the funding body. 
F. V. Z. conceptualised and designed the study, supervised data collection, drafted the initial manuscript and reviewed and revised the manuscript. N. O. collected data and samples, carried out the laboratory analyses of samples and reviewed the manuscript. R. A. S. coordinated data analysis and critically reviewed the manuscript for important intellectual content. R. A. V. co-supervised data collection and reviewed and revised the manuscript. A. M. conceptualised and designed the study, co-supervised data collection and reviewed and revised the manuscript. All authors approved the final manuscript as submitted and agree to be accountable for all aspects of the work

The authors declare that there are no conflicts of interest.

\section{References}

1. Whitford GM (1994) Intake and metabolism of fluoride. Adv Dent Res 8, 5-14.

2. Palmer C \& Wolfe SH, American Dietetic Association (2005) Position of the American Dietetic Association: the impact of fluoride on health. I Am Diet Assoc 105, 1620-1628.

3. Frencken JE, Sharma P, Stenhouse L, et al. (2017) Global epidemiology of dental caries and severe periodontitis - a comprehensive review. J Clin Periodontol 44, Suppl. 18, S94-S105.

4. Szpunar S \& Burt B (1990) Fluoride exposure in Michigan schoolchildren. J Public Health Dent 50, 18-23.

5. Pendrys DG \& Stamm JW (1990) Relationship of total fluoride intake to beneficial effects and enamel fluorosis. J Dent Res 69, 529-538 (discussion 556-557).

6. Warren JJ, Levy SM \& Kanellis MJ (2001) Prevalence of dental fluorosis in primary dentition. J Public Health Dent 61, 87-91.

7. Hong L, Levy SM, Broffitt B, et al. (2006) Timing of fluoride intake in relation to development of fluorosis on maxillary central incisors. Community Dent Oral Epidemiol 34, 299-309.

8. Institute of Medicine (1997) Dietary Reference Intakes for Calcium, Magnesium, Vitamin D, and Fluoride. Washington, DC: National Academies Press.

9. National Health and Medical Research Council and Ministry of Health (2017) Nutrient reference values for Australia and New Zealand including recommended dietary intakes: fluoride (updated 2017) version 1.1. https://www.nrv.gov.au/sites/ default/files/content/Fluoride\%20section.pdf (accessed September 2018).

10. Zohoori FV, Whaley G, Moynihan PJ, et al. (2014) Fluoride intake of infants living in non-fluoridated and fluoridated areas. Br Dent $J \mathbf{2 1 6}, \mathrm{E} 3$.

11. Maguire A \& Zohoori FV (2013) Fluoride balance in infants and young children in the UK and its clinical relevance for the dental team. Br Dent J 214, 587-593.

12. Zohoori FV \& Maguire A (2016) Development of a database of the fluoride content of selected drinks and foods in the UK. Caries Res 50, 331-336.

13. Butte NF (2005) Energy requirements of infants. Public Health Nutr 8, 953-967.

14. Scientific Committee on Food (2003) Report of the Scientific Committee on Food on the Revision of Essential Requirements of Infant Formulae and Follow-on Formulae. Brussels: European Commission Health and Consumer Protection Directorate-General.

15. Zohouri FV, Swinbank CM, Maguire A, et al. (2006) Is the fluoride/creatinine ratio of a spot urine sample indicative of 24-h urinary fluoride? Community Dent Oral Epidemiol 34, $130-138$.
16. Martínez-Mier EA, Cury JA, Heilman JR, et al. (2011) Development of gold standard ion-selective electrode-based methods for fluoride analysis. Caries Res 45, 3-12.

17. Thway TM, Eschenberg M, Calamba D, et al. (2011) Assessment of incurred sample reanalysis for macromolecules to evaluate bioanalytical method robustness: effects from imprecision. AAPS J 13, 291-298.

18. Cumming G (2012) Understanding the New Statistics: Effectsizes, Confidence Intervals and Meta-analysis. New York: Routledge Academic.

19. Lakens D (2013) Calculating and reporting effect sizes to facilitate cumulative science: a practical primer for $t$-tests and ANOVAs. Front Psychol 4, 863.

20. Cohen J (1988) Statistical Power Analysis for the Behavioral Sciences. New York: Routledge Academic.

21. Bergman C, Gray-Scott D, Chen JJ, et al. (2009) What is next for the dietary reference intakes for bone metabolism related nutrients beyond calcium: phosphorus, magnesium, vitamin D, and fluoride? Crit Rev Food Sci Nutr 49, 136-144.

22. World Health Organization (2010) Inadequate or Excess Fluoride: A Major Public Health Concern. Geneva: World Health Organization.

23. World Health Organization (2014) Basic Methods for Assessing Renal Fluoride Excretion in Community Prevention Programmes for Oral Health. Geneva: World Health Organization.

24. Spak CJ, Hardell LI \& De Chateau P (1983) Fluoride in human milk. Acta Paediatr Scand 72, 699-701.

25. Ekstrand J, Boreus LO \& de Chateau P (1981) No evidence of transfer of fluoride from plasma to breast milk. Br Med J (Clin Res Ed) 283, 761-762.

26. Ekstrand J, Spak CJ, Falch J, et al. (1984) Distribution of fluoride to human breast milk following intake of high doses of fluoride. Caries Res 18, 93-95.

27. Ekstrand J, Hardell LI \& Spak CJ (1984) Fluoride balance studies on infants in a 1-ppm-water-fluoride area. Caries Res 18, 87-92.

28. Levy SM, Warren JJ, Davis CS, et al. (2001) Patterns of fluoride intake from birth to 36 months. J Public Health Dent 61, $70-77$.

29. Barnett HL (1950) Kidney function in young infants. Paediatrics 5, 171-179.

30. Sulemanji M \& Vakili K (2013) Neonatal renal physiology. Semin Pediatr Surg 22, 195-198.

31. Shen YW \& Taves DR (1974) Fluoride concentrations in human placenta and maternal and cord blood. Am J Obstet Gynecol 119, 205-207.

32. Ekstrand J \& Ehrnebo M (1979) Influence of milk products on fluoride bioavailability in man. Eur J Clin Pharmacol 16, 211-215.

33. Zohoori FV (2018) Summary of general discussion and conclusions. Adv Dent Res 29, 183-185.

34. Ekstrand J, Ziegler EE, Nelson SE, et al. (1994) Absorption and retention of dietary and supplemental fluoride by infants. $A d v$ Dent Res 8, 175-180.

35. Villa A, Anabalon M, Zohouri V, et al. (2010) Relationships between fluoride intake, urinary fluoride excretion and fluoride retention in children and adults: an analysis of available data. Caries Res 44, 60-68.

36. Ekstrand J, Fomon SJ, Ziegler EE, et al. (1994) Fluoride pharmacokinetics in infancy. Pediatr Res 35, 157-163.

37. Berg J, Gerweck C, Hujoel PP, et al. (2011) Evidence-based clinical recommendations regarding fluoride intake from reconstituted infant formula and enamel fluorosis: a report of the American Dental Association Council on Scientific Affairs. J Am Dent Assoc 142, 79-87. 